\title{
Ruptured Abdominal Aortic Aneurysm Associated with Left Colon Gangrene and Neurological Disorders, a Very Rare and Life-Threatening Combination
}

\author{
Sokol Xhepa ${ }^{1 *}$, Saimir Kuci ${ }^{3}$, Myzafer $\mathrm{Kaci}^{2}$, Gentian Caco ${ }^{1}$, Petrika Gjergo ${ }^{1}$, \\ Stavri Llazo ${ }^{3}$, Ervin Bejko ${ }^{3}$, Alfred Ibrahimi ${ }^{3}$, Frenki Vila²
}

Received: 16 April 2021 / Accepted: 07 May 2021 / Published online: 20 July 2021

This article is published with open access at https://journal.astes.org.al

(C) The author(s) 2021. \& Copyright (C) 2021, the Albanian Society for Trauma and Emergency Surgery

(c) The Albanian Journal of Trauma and Emergency Surgery is an Open Access Journal. All articles are distributed under the terms of the Creative Commons Attribution Non-Commercial License: http://creativecommons.org/licenses/by-nc/4.0/) which permits unrestricted non-commercial use, distribution, and reproduction in any medium provided the original work is properly cited.

\begin{abstract}
Background: We report the management of a very rare combination of two severe surgical emergencies, ruptured aortic aneurysm with gangrene of the left colon. Both events separately present a high mortality rate in ruptured aortic aneurysms with $48.5 \%$.

Case report: We present the case of a 59-year-old woman that was admitted to the service of Emergencies at University Hospital Center "Mother Theresa" of Tirana on January 4th of 2013, after being transferred from the Regional Hospital of Durres diagnosed with ruptured aortic aneurysm based on an unclear CT. The patient arrived in a state of profound hypovolemic shock after suffering cardiac arrest and underwent resuscitation at the ICU of Durres Hospital ( no exact information of the anoxic brain time). After a brief volemic compensation in our ICU, the patient was taken to the operating room. Intraoperatively we found an infrarenal $r$ AAA and gangrene of the sigmoid and left colon. Resection and reconstruction by the interposition of a tube graft were performed, followed by a left large hemicolectomy with temporary colostomy realized by the team of general surgeons. Three months later they performed colorectal-anastomosis as a second step operation.

Postoperatively the patient had a period of 3 weeks stay in the ICU, mostly due to neurological complications after the ischemic stroke, and on January 29 of 2013, she returned to the Service of Neurology at the Hospital of Durres for further neurological rehabilitation. One year later, in the ambulatory routine check, the patient presented full recovery from surgery and a complete central and peripheral neurological rehabilitation.

Conclusions: Ruptured aortic aneurysm with gangrene of the left colon is a very rare and severe combination caused by the hypoperfusion of the inferior mesenteric artery in the presence of hypovolemic shock and insufficient collateral circulation. The strategy of treatment includes fast diagnosis, short hypotensive resuscitation, cell-saving and autotransfusion, and the simplest possible surgical reconstruction for both emergencies.
\end{abstract}

Keywords: Ruptured, aortic aneurysm, gangrene, colon, acute abdomen

Original article, no submission or publication in advance or in parallel

\section{* Corresponding author:}

Asc. Prof. Dr. Sokol Xhepa M D, Ph.D

\mdsxhepa@yahoo.com

1 Service of Angiology and Vascular Surgery, University Hospital Centre "Mother Theresa" of Tirana, ALBANIA

2 Service of General Surgery, University Hospital Centre "Mother Theresa" of Tirana, ALBANIA.

3 Service of Anesthesiology and Intensive Care, University Hospital Centre "'Mother Theresa" of Tirana, ALBANIA.

\section{Introduction}

The ruptured aortic aneurysm (rAAA) is an abdominal aortic aneurysm (AAA) with extraluminal blood on computed tomography (CT) or noted clinically at the time of surgery. A contained rupture refers to blood outside the aneurysm sac that is confined to the retroperitoneal space, tamponaded by the surrounding tissues. A free rupture refers to bleeding into the peritoneal cavity, without tamponade. A symptomatic, non-ruptured AA is one with back pain or tenderness over the aorta on deep palpation but with an intact aneurysm on CT. The pain is thought to be related to 
acute expansion of the wall, intramural hemorrhage, wall degeneration, or bleeding into the thrombus and is therefore considered a prelude to actual rupture, which requires urgent repair. Symptomatic aneurysms are not associated with hypotension; however, the prognosis is much better than that of rAAA (but worse compared to selective repair).

Regarding the mortality rate of rAAA, data from the Danish national registry from 1994 to 2008 show the overall mortality risk from rAAA to be $76 \%$ (less than half underwent surgery). Postoperative mortality decreased from $51 \%$ to $42 \%$. This was attributed to centralization of services and prehospital care. The most ambitious review of population data was by Mani et al, using the Vascunet database. In 2011, they collated outcomes for rAAA repair in 7040 patients from national and regional registries in nine countries (Australia, Denmark, Finland, Hungary, Italy, Norway, Sweden, Switzerland, and the United Kingdom). The overall mortality rate was $31.6 \%$, but it declined by $4.2 \%$ between 2005 and 2009.

A meta-analysis of the first 50 years of rAAA publications noted that the mortality rate for ruptured repair has fallen only $3.5 \%$ per decade since the initial successful repairs were reported.

The high postoperative mortality rates after aortic rupture are secondary to a high incidence of myocardial infarction, renal failure, and multiple organ failure (MOF). Aortic rupture and OSR result in a combination of ischemiareperfusion injuries, hemorrhagic shock and lower torso ischemia.

\section{Clinical diagnosis:}

The classic presentation of rAAA is a patient older than 60 years complaining of acute-onset abdominal and back pain. Back pain is caused by rupture of the aneurysm into the retroperitoneal space and is severe and unremitting. Patients may describe pain radiating to the testes, inguinal canal, or rectum. Some patients may describe hip pain due to psoas irritation. On examination, the patient is usually pale, diaphoretic, and hypotensive with a tender, expansile abdominal mass. Rarely, there may be flank ecchymosis due to tracking of retroperitoneal blood. There may be documentation of a prior diagnosis of AAA.

\section{Our case report:}

We present a very exceptional case of a 59-year-old female patient, with no comorbidities, that was admitted in the regional hospital of Kavaja in the early hours of the day, on the 4th of January in 2013. Symptoms onset with severe abdominal pain and hypotension, thus at the fist inspection classified as an acute abdomen. After having briefly received the first line medical support and physical/clinical examination the patient was immediately transferred to the regional hospital of Durres for further examination and possible intervention. A CT scan with no intravenous contrast was performed briefly from the arrival into the hospital of Durres. The image of the CT suggested a giant aneurysm of the abdominal aorta. The vital parameters of the patients suffered a further deterioration followed by cardiac arrest. The ICU team therefore performed cardiopulmonary resuscitation succeeding to restore spontaneous blood circulation and breathing. After intensive monitoring and careful fluid redistribution the patient was transferred to Mother Theresa Hospital in Tirana.

The rAAA is a vascular surgical emergency, and when the diagnosis is being considered, immediate vascular surgery consultation is critical. The patient manifested severe hemorrhagic hypotension and no peripheral pulse in the clinical examination.

Permissive Hypotension: Preoperative resuscitation of rAAA patients with hypotension must be judicious. Large volumes of intravenous fluids and a rise in blood pressure can lead to further hemorrhage by overcoming the tamponade that stabilized the initial rupture. It also causes hemodilution, coagulopathy, hypothermia, and acidosis, and further deterioration. Similar to transfusion protocols that restrict aggressive fluid resuscitation in hypotensive trauma patients with hemorrhagic shock, permissive hypotension for rAAA patients is recommended.

The patient underwent one unnecessary stretch in time (meaning difficulties in decision making from the family members), adding up to a period of 5 hours preoperatory time. In a study conducted in Belgrade (referenced at the bottom of this print), it is concluded that the optimal gap of time to intervene between the rupture of the blood vessel and the operatory room is 30 minutes. Every following minute is a detrimental factor in the influence of early survival.

Intraoperatively an infrarenal aneurysm confined by a giant retroperitoneal hematoma was located after exposing the retroperitoneum and adjacent to the latter a very obvious discoloration of the descendent part of the colon with inferior delimitation into the recto sigmoidal junction. The ischemic damage of the intestine had evolved into transmural damage followed by gangrene.

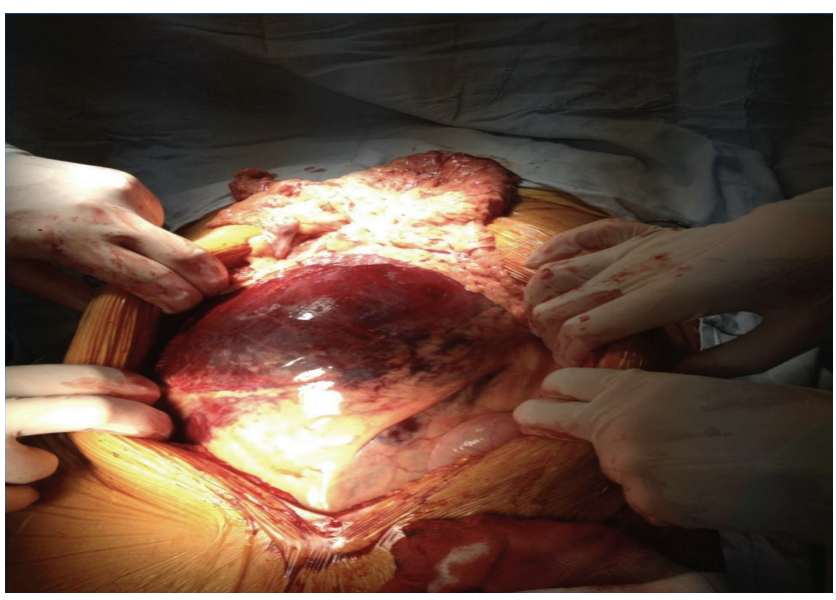

Figure 1. View of the intestines at the moment of opening the abdomen. 
The operating team of vascular surgeons performed surgery of the primary and more acute life-threatening condition. Following laparotomy, the presence of a very large retroperitoneal hematoma needed a rapid control that was achieved by placement of a supraceliac clamp for 5-10 min, (fig. 1) followed by a repositioned infrarenal clamp after the aneurismatic neck of the infrarenal aorta could be located properly. The distal control was achieved through placement of iliac clamps. Once the aneurysm sac was opened, retrograde bleeding from the inferior mesenteric and residual lumbar arteries is controlled by suture ligation. The Aortic repair was accomplished rapidly with a tube graft, (fig. 2) given the current state of the iliac vessels. Proximal and distal clamps are removed and the revascularization of the great vessel was obtained.

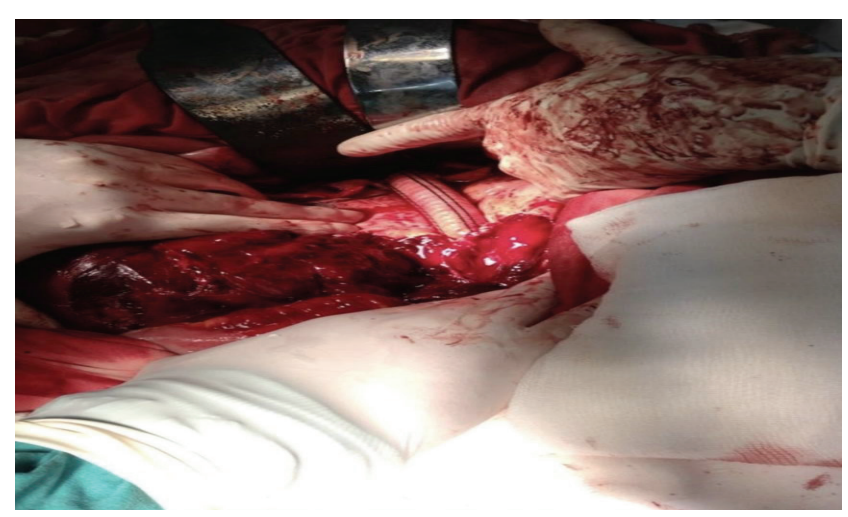

Figure 2. Placement of the vascular graft at the level of the iliac vessels

The descendent and sigmoid colon showed no signs of reperfusion reinforcing the diagnosis of transmural gangrene (fig. 3).

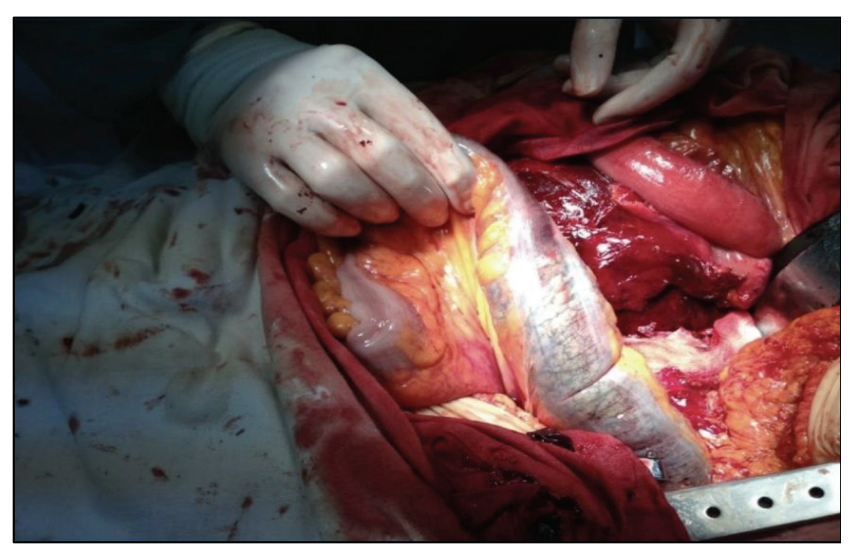

Figure 3. Inspection of the colonic segment

The general surgery team joined the intervention. Inspection of the colonic segment is carefully done. The conclusion was to perform a large left hemicolectomy with resection into safe borders of colored and vascularized colon and the placement of a temporary stomy. We should mention that in such cases the simplest possible surgical treatment is the most lifesaving.
The patient was treated for 3 weeks in the ICU. Postoperatively the patient developed temporary renal insufficiency. The Kidney dysfunction in rAAA patients has long been recognized to have a significant deleterious impact on mortality. Although the incidence of renal dysfunction after elective AAA repair is relatively low, rAAA patients have a significantly higher incidence of experiencing renal dysfunction that can range from $26 \%$ to $42 \%$. In addition the patient manifested neurological complications linked to the ischemia timing that followed the cardiac arrest, as it will be expanded in more detail in the discussion session of this article. A rich collaboration with the neurologists ensured the rehabilitation of the central and peripheral stroke complications that were further treated in the Neurology unit in Durres where she was transferred on the $25^{\text {th }}$ of January. Three months after the $1^{\text {st }}$ surgery the reconstruction of the intestinal transit was successfully performed, through the termino-terminal anastomosis of the transverse colon and the upper rectal segment. In July 2013 during the ambulatory visit the patient (fig. 4) presented a total recovery of neurological functions and a return to normal of the usual lifestyle.

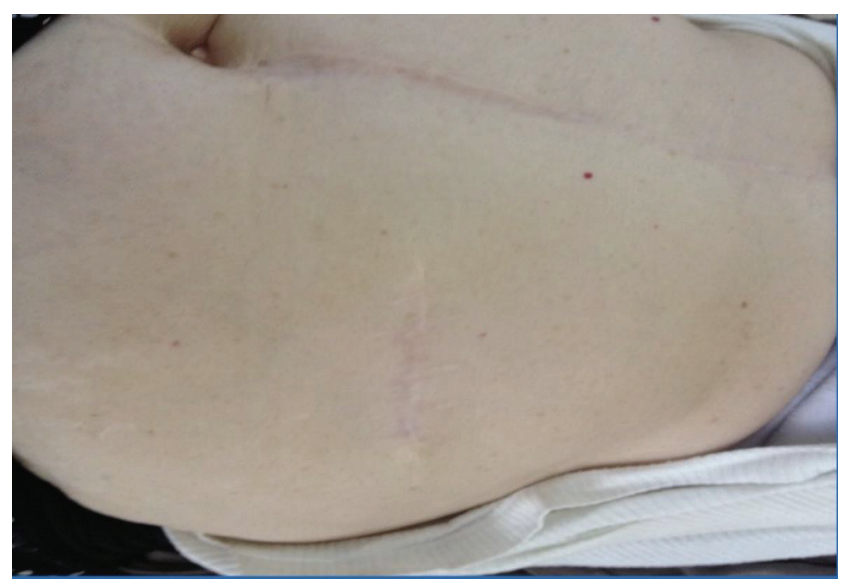

Figure 4. Operative wound after intestinal reconstruction

\section{Discussions:}

Our case presented a very rare form of clinical and interoperate presentation that hypothetically was linked to the hypoperfusion state post CA and to the vascular supply of the ischemic segment of the colon. Even though colonic ischemia following the procedure of aorta repair is not an uncommon complication, the simultaneous finding of rAAA and colonic ischemia (gangrenous state) in the laparotomy suggests of a hypoperfusion ischemia.

Non occlusive mesenteric ischemia is characterized by signs of intestinal hypoperfusion without evidence of mechanical obstruction of a supplying vessel. Left untreated it can lead to often extensive necrosis which is associated with a poor prognosis even when treated rapidly. The pathophysiology of this condition is incompletely understood, but low cardiac output, for example, after 
cardiogenic shock, and the use of vasoconstricting agents are associated. In patients suffering from cardiac arrest, circulation comes to a stop within seconds, leading to loss of consciousness and progressive ischemia. The treatment of choice is the immediate start of cardiopulmonary resuscitation (CPR). Measurements in animal models of CPR show that cardiac output is restored to less than $40 \%$ of pre-arrest values during mechanical compressions. Furthermore, gastrointestinal perfusion is highly impaired in the period after the return of spontaneous circulation (ROSC), presumably in an attempt to preserve oxygen for the brain and heart. When CPR is successful and ROSC is achieved, sudden reperfusion of ischemic areas leads to the release of cytokines and a generalized inflammatory response. This ischemia reperfusion injury causes additional damage in the digestive tract. The cardiac output remains impaired for some time, likely due to myocardial stunning.

Another factor we should take into consideration is the vascular anatomy of the affected region of the colon and its process to ischemia. The collateral flow through the mesenteric branches is provided via the marginal artery of Drummond and the meandering mesenteric artery, also known as the Arc of Riolan (AoR). The marginal artery runs parallel to the colon to give branches to the vasa recta.

The marginal artery runs along the splenic flexure, but is absent or underdeveloped in $5 \%$ of the population. Injury to the colon is believed to typically occur in the "watershed" areas of the splenic flexure (Grifiths point) and sigmoid colon (Sudeck point). (fig. 5)

The pathophysiology of ischemic colitis is more often an acute, self-limited decrease in blood supply rather than a specific vascular lesion or embolic event. Angiography, when abnormal, shows narrowing of the small vessels and tortuosity of the long colonic arteries.

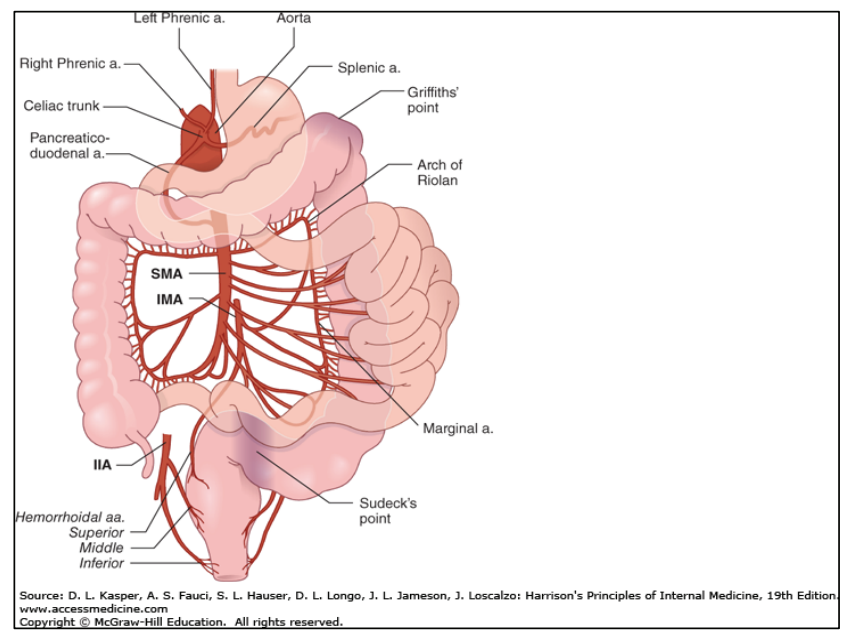

Figure 5. Blood Supply Of The Colon .

Within a fifteen-minute window a reduction in blood flow leading to ischemia can demonstrate structural changes in the mucosa. After three hours mucosal sloughing will be evident and after six hours transmural necrosis will manifest.
There are two main factors that cause structural damage, (I) hypoxia, due to a reduction in blood flow and (II) reperfusion injury.

Therefore, colonic insult in these districts results from diminished blood flow. This can range from superficial injury to the mucosa and submucosal layer to full thickness necrosis of the colonic wall, as was the case of our patient resulting to gangrene and necrosis of the colon. Immediate respective surgery was unenviable.

The cerebral damage is also linked to the hypoperfusion state during and following CA hypovolemic shock state of the patient. (fig. 6)

The hypoxic ischemic brain injury (HIBI) after cardiac arrest (CA) is a leading cause of mortality and long-term neurologic disability in survivors. The pathophysiology of HIBI encompasses a heterogeneous cascade that culminates in secondary brain injury and neuronal cell death. This begins with primary injury to the brain caused by the immediate cessation of cerebral blood flow following CA. Thereafter, the secondary injury of HIBI takes place in the hours and days following the initial $\mathrm{CA}$ and reperfusion. Among factors that may be implicated in this secondary injury include reperfusion injury, microcirculatory dysfunction, impaired cerebral autoregulation, hypoxemia, hyperoxia, hyperthermia, fluctuations in arterial carbon dioxide, and concomitant anemia.

HIBI is associated with significant neurologic disability, ranging from mild cognitive deficits to minimally conscious and persistent vegetative states.

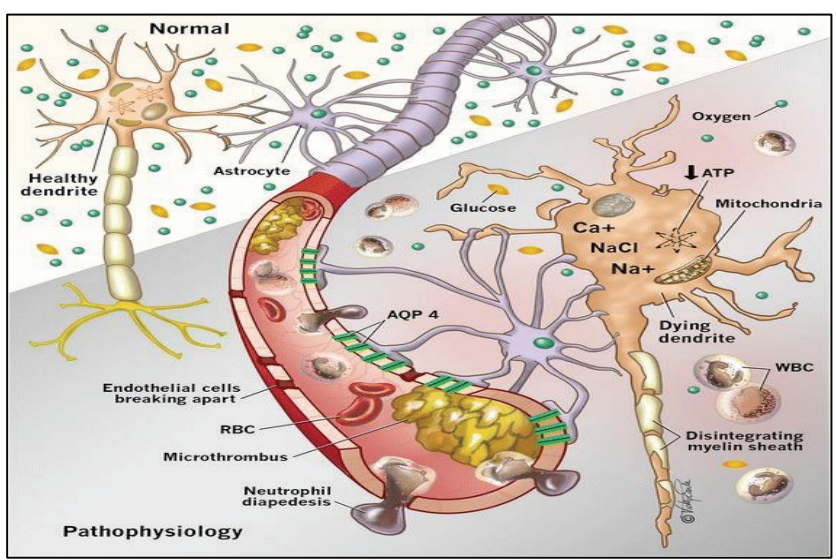

Figure 6. Pathophysiology of hypoperfusion state

A schematic demonstrating the various microvascular and cellular pathophysiologic consequences which occur during the primary and secondary injury in hypoxic ischemic brain injury (HIBI). Decreased cerebral oxygen delivery manifests as reduced neuronal aerobic metabolism, causing reduced cellular adenosine triphosphate (ATP) production. Intracellular calcium accumulation leads to mitochondrial toxicity and further reduced ATP production. Inability to sustain cellular respiration results in cell death and apoptosis. Additionally, in the microvasculature, endothelial dysfunction leads to a porous blood-brain barrier, formation 
of cerebral edema, formation of microthrombi and limitation of cerebral blood flow with exacerbation of cellular ischemia. AQP 4 Aquaporin-4, RBC Red blood cells, WBC White blood cells.

\section{Conclusions}

Considering the tirade of pathogenically insults of rAAA, Colonic Ischemia and hypoxic ischemic brain injury), we must consider the clinical case as a very rare one, followed by a very high mortality rate. Even considered apart the three complications represent a high mortality rate. The colonic ischemia post rAAA graft repair, is not an uncommon complication due to the diminished vascularization in the Griffith point and in the Suddeck point, representing the most common points of the left colon affected from the changes of blood flow. Regarding our case we came into the conclusion that it was linked to the hypoperfusion that followed the cardiac arrest and continued through the preoperatory period. Literature and interoperate logic do not suggest for the operation and the inferior mesenteric a. ligation to be the cause that leaded into colonic ischemia (rAAA and colonic ischemia were simultaneous findings during laparotomy).

It should be taken into consideration that colonic ischemia is a dreaded complication following AAA repair. Although branches from the celiac, SMA, IMA, hypogastric, and profunda femoris arteries all converge to form a network of collaterals that supply the colon, altered vascular anatomy following vascular reconstruction, combined with preexisting atherosclerotic disease places patients at potential increased risk for ischemia. Ligation of the IMA, failure to revascularize the hypogastric arteries, preexisting iliofemoral occlusive disease, SMA stenosis, as well as atheroembolism, retractor injury, and previous colonic resection, can all contribute to colonic ischemia, which has a clinical incidence $0.2 \%$ to $6 \%$ following open surgery.

Neurologic complications remain strictly linked to the hyper perfusion state as discussed above. The collaboration we shared with the neurologists helped to gain a full rehabilitation of the peripheral and central stroke complications.

And last but not least aside from the surgical team's combined effort and successful surgery, the young age and the absence of comorbidities are 2 positive factors in the lead of the uneventful recovery of this patient.

The rAAA is a vascular emergency, and immediate vascular surgery consultation is critical. The hospitals that lack vascular surgery expertise and cannot offer definitive treatments should consider establishing networks with larger institutions that have vascular surgery services and can offer repair by endovascular or open surgical means, as needed. The impact of transferring rAAA patients to regional centers where repair can be carried out by experienced vascular teams has been studied. Rural hospitals without vascular surgery expertise tend to face a higher burden of rAAA patients, and national trends suggest that they are more likely to transfer patients to regional centers. Although patient transfer might increase the time of arrival from the initial hospital to the operating room, most studies suggest that high-volume regional centers significantly lower the mortality for rAAA patients.

COI Statement: This paper has not been submitted in parallel. It has not been presented fully or partially at a meeting or podium or congress. It has not been published nor submitted for consideration beforehand.

This research received no specific grant from any funding agency in the public, commercial, or non-profit sectors. There are no relevant or minor financial relationships from authors, their relatives or next of kin with external companies.

Disclosure: The authors declared no conflict of interest. No funding was received for this study.

Acknowledgement: We would like to thank medical staff of. Service of Angiology, Vascular Surgery and General Surgery University Hospital Centre "Mother Teresa", Tirana, ALBANIA.

\section{References:}

1. Turk KA: The post-mortem incidence of abdominal aortic aneurysm. Proc R Soc Med 58(Part 1):869-870, 1965.

2. Armour RH: Survivors of ruptured abdominal aortic aneurysm: the iceberg's tip. Br Med J 2:1055-1057, 1977.

3. Bown MJ, et al: A meta-analysis of 50 years of ruptured abdominal aortic aneurysm repair. Br J Surg 89:714-730, 2002.

4. Schermerhorn ML, et al: Changes in abdominal aortic aneurysm rupture and short-term mortality, 1995-2008: a retrospective observational study. Ann Surg 256:651-658, 2012.

5. Norman PE, et al: Falling rates of hospitalization and mortality from abdominal aortic aneurysms in Australia. J Vasc Surg 53:274-277, 2011

6. Ascher E, et al: Ruptured versus elective abdominal aortic aneurysm repair: outcome and cost. Ann Vasc Surg 13:613617, 1999

7. Muschitz, GK, Fochtmann, A, Keck, M. Non-occlusive mesenteric ischemia: The prevalent cause of gastrointestinal infarction in patients with severe burn injuries. Injury 2015; 46: 124-130.

8. Trompeter, M, Brazda, T, Remy, CT. Non-occlusive mesenteric ischemia: Etiology, diagnosis, and interventional therapy. Eur Radiol 2002; 12: 1179-1187.

9. Bailey, RW, Bulkley, GB, Hamilton, SR. Protection of the small intestine from nonocclusive mesenteric ischemic injury due to cardiogenic shock. Am J Surg 1987; 153: 108-116.

10. Ludwig, KA, Quebbeman, EJ, Bergstein, JM. Shockassociated right colon ischemia and necrosis. J Trauma 1995; 39: 1171-1174. 
11. Levandoski, G, Deitrick, JE, Brotman, S. Necrosis of the colon as a complication of shock. Am Surg 1988; 54: 621-626.

12. Sitges-Serra, A, Mas, X, Roqueta, F. Mesenteric infarction: An analysis of 83 patients with prognostic studies in 44 cases undergoing a massive small-bowel resection. Br J Surg 1988; 75: $544-548$.

13. Ascher E, et al: Ruptured versus elective abdominal aortic aneurysm repair: outcome and cost. Ann Vasc Surg 13:613617, 1999.

14. Bown MJ, et al: A meta-analysis of 50 years of ruptured abdominal aortic aneurysm repair. Br J Surg 89:714-730, 2002.

15. Schermerhorn ML, et al: Changes in abdominal aortic aneurysm rupture and short-term mortality, 1995-2008: a retrospective observational study. Ann Surg 256:651-658, 2012.
16. Maybury RS, et al: Rural hospitals face a higher burden of ruptured abdominal aortic aneurysm and are more likely to transfer patients for emergent repair. J Am Coll Surg 212:1061-1067, 2011.

17. Holt PJ, et al: Meta-analysis and systematic review of the relationship between volume and outcome in abdominal aortic aneurysm surgery. Br J Surg 94:395-403, 2007

18. Belgrade - 30 minutes, Davidovic L - rAAA: Factors Influencing Early Survival - Ann Vasc Surg 2005, 19(1), 29-33

19. Politoske EJ-Am J Gastroenterol: rAAA presenting as an obstruction of the left colon. 1990 Jun 85(6) 745-7.

20. Stenberg B: AAA complicated by rectal necrosis. An unusual complication - Acta Chir Scand 1984, 150(6) 497-8. 